\title{
Simulation technique for hard-disk models in two dimensions
}

Fraser, Diane P.; Zuckermann, Martin J.; Mouritsen, Ole G.

Published in:

Physical Review A

Link to article, DOI:

10.1103/PhysRevA.42.3186

Publication date:

1990

Document Version

Publisher's PDF, also known as Version of record

Link back to DTU Orbit

Citation (APA):

Fraser, D. P., Zuckermann, M. J., \& Mouritsen, O. G. (1990). Simulation technique for hard-disk models in two dimensions. Physical Review A, 42(6), 3186-3195. https://doi.org/10.1103/PhysRevA.42.3186

\section{General rights}

Copyright and moral rights for the publications made accessible in the public portal are retained by the authors and/or other copyright owners and it is a condition of accessing publications that users recognise and abide by the legal requirements associated with these rights.

- Users may download and print one copy of any publication from the public portal for the purpose of private study or research.

- You may not further distribute the material or use it for any profit-making activity or commercial gain

- You may freely distribute the URL identifying the publication in the public portal

If you believe that this document breaches copyright please contact us providing details, and we will remove access to the work immediately and investigate your claim 


\title{
Simulation technique for hard-disk models in two dimensions
}

\author{
Diane P. Fraser and Martin J. Zuckermann \\ Department of Physics and Centre for the Physics of Materials, McGill University, \\ 3600 University Street, Montréal, $P Q$, Canada H3A $2 T 8$ \\ Ole G. Mouritsen \\ Department of Structural Properties of Materials, The Technical University of Denmark, Building 307, DK-2800 Lyngby, Denmark \\ (Received 2 April 1990)
}

\begin{abstract}
A method is presented for studying hard-disk systems by Monte Carlo computer-simulation techniques within the $N p T$ ensemble. The method is based on the Voronoi tesselation, which is dynamically maintained during the simulation. By an analysis of the Voronoi statistics, a quantity is identified that is extremely sensitive to structural changes in the system. This quantity, which is derived from the edge-length distribution function of the Voronoi polygons, displays a dramatic change at the solid-liquid transition. This is found to be more useful for locating the transition than either the defect density or the pressure-area isotherm.
\end{abstract}

\section{INTRODUCTION}

The two-dimensional hard-disk fluid has been a seminal model in the development of the theories of liquids and liquid structure. Since it is well established ${ }^{1-3}$ that this model undergoes an order-disorder transition, it can be considered as a minimal nonlattice model exhibiting a cooperative phenomenon. For convenience we refer to this order-disorder transition as a solid-liquid transition. Despite the simplicity of the hard-disk model, the statistical mechanics of the model has not as yet been solved exactly, though a large number of numerical and approximate analytical calculations have been carried out. Indeed, the Monte Carlo simulation technique was pioneered within statistical mechanics ${ }^{4}$ by a calculation of the equation of state of the two-dimensional hard-disk model. The present status of theoretical knowledge about the model does not appear to have been reviewed recently (Ref. 5 contains a review of nonhard systems). Since this knowledge is a prerequisite for the development of the present paper, we first present a brief overview of the current status of theoretical work for twodimensional hard-core fluids.

The two-dimensional hard-disk fluid is one of the simplest nonlattice systems to study by computer-simulation techniques. Metropolis et al. ${ }^{4}$ first used the Monte Carlo (MC) simulation technique to study a two-dimensional system of 224 hard disks with periodic boundary conditions. Their results were in agreement with free-volume theory at high densities and with a four-term virial expansion at lower densities. Molecular-dynamics (MD) methods were used by Alder and Wainwright ${ }^{1}$ on a system of 870 hard disks and by Hoover and Alder, ${ }^{2}$ on smaller systems, to study the liquid-solid transition and the effect of system size. The isotherms for the larger systems showed signs of a van der Waals-like loop at the phase transition. A shift in transition pressure was attributed to the communal entropy of the systems. Monte Carlo simulation techniques in the isothermal-isobaric
$(N p T)$ ensemble were developed by Wood $^{3,6}$ and applied initially to a system of 12 hard disks. Larger systems gave results in agreement with those of molecular dynamics $^{1,2}$ and constant-NVT Monte Carlo studies. ${ }^{7}$

Analytical integral equation techniques (see, for example, Ref. 8 for a review) are, so far, unable to describe the liquid-solid transition. They can be used with success, though, in the liquid and solid phases away from the transition. Chae, Ree, and $\mathrm{Ree}^{7}$ applied several integral equations to radial distribution functions obtained from Monte Carlo simulations and concluded that a modified Born-Green-Yvon equation yielded better results than the Percus-Yevick equation used in conjunction with the virial theorem. Comparable with the Percus-Yevick equation was the scaled particle theory (SPT) equation of Helfand, Frisch, and Lebowitz. ${ }^{9}$ Cotter and Stillinger ${ }^{10}$ extended the SPT equation to give better agreement at higher densities. The expression they obtained though is complicated and the integrals have to be solved numerically. Henderson ${ }^{11}$ compared the expansion of the SPT equation with the first six virial coefficients and modified the SPT equation (SPTH) to give even closer agreement with the virial expansion. $\mathrm{He}$ made a further modification $^{12}$ and then compared this with MC data from a small system of 32 particles as well as with some of the simulation data of other workers., ${ }^{3,13-15}$ The MC data were then used as a base for a perturbation theory of the two-dimensional Lennard-Jones fluid. Andrews ${ }^{16}$ developed an analytic equation of state for the liquid phase based on the first three virial coefficients. This gave better agreement with molecular-dynamics data ${ }^{2}$ than did the 7 -series virial expansion, ${ }^{17}$ though it was not as good as the SPT equation. However, a numerical solution based on the first four coefficients gave an improvement over SPT. The method was also applied to the hard-disk crystal phase with reasonable success, differing from molecular-dynamics results ${ }^{18}$ by less than $10 \%$ right up to 0.9 of the close-packed density, and to systems with attractive wells and Lennard-Jones potentials. The main 
improvement by the work of Andrews is that the equations of state give a singularity at the correct closepacked limit to the packing fraction $\left(\eta_{0}=\pi / 2 \sqrt{3} \sim 0.9069\right)$. The SPT equations all give a limiting packing fraction of unity. $\mathrm{Kratky}^{19}$ improved upon the sixth and seventh virial coefficients and estimated the subsequent three coefficients $\left(B_{8}, B_{9}\right.$, and $\left.B_{10}\right)$, using extrapolation techniques applied to Padé approximants, an extension of Andrew's method based on five virial coefficients, and a modified version of Henderson's SPTH equation. More recently, Erpenbeck and Luban ${ }^{20}$ have performed combined MC-MD simulations on harddisk systems in the fluid phase and found excellent agreement with results obtained by applying a Levin approximant to the first six terms of the virial series.

Recent two-dimensional studies have also concentrated on anisotropioc hard particles. These include simulations of hard ellipses, ${ }^{21}$ discorectangles, ${ }^{22}$ needles, ${ }^{23}$ and dumbbells. $^{24}$ Theoretical equations of state have also been developed in parallel with the simulation studies. Several workers extended the SPT equation of state to planar convex particles ${ }^{25,26}$ and dumbbells ${ }^{27}$ and the Percus-Yevick equation has been solved numerically for ellipses ${ }^{28}$ and dumbbells. ${ }^{29}$

In this paper we present the results from constant $N p T$ Monte Carlo simulations of two-dimensional hard-disk systems which use a new technique, based on the Voronoi tesselation $^{30}$ of the system, to keep track of nearest neighbors. The computing time used is shown in Sec. II to be proportional to the number of particles in the system, even for small system sizes. Usual simulation methods, which make use of lists of neighbors, can run in linear time, if the lists are not updated too often, but for low densities or small system sizes $(N \sim 100)$ the time is usually quadratic.

We will first describe the technique and then show how an analysis of the Voronoi tesselation may be used to isolate the liquid-solid tranasition in single species hard-disk systems. This analysis is based on the edge-length distribution function of the Voronoi polygons. We find that a particular decomposition of this distribution function provides a strong signal of the liquid-solid transition which is superior to the signal seen in the pressure-area $(p-A)$ isotherm or properties like the defect density.

\section{SIMULATIONS}

\section{A. Constant $N p T$ Monte Carlo simulations}

The starting configurations for the simulations were obtained using the method employed previously by Fraser et al. ${ }^{31}$ which will be described in Sec. II B 1.

Particle moves were performed using the standard Metropolis procedure. ${ }^{4} \mathrm{~A}$ particle was first chosen at random. A random displacement of the particle $(\delta x, \delta y)$ was then considered where

$$
\begin{aligned}
& \delta x=\left(2 \zeta_{x}-1\right) \delta r_{\max }, \\
& \delta y=\left(2 \zeta_{y}-1\right) \delta r_{\max },
\end{aligned}
$$

with random numbers $\zeta_{x}$ and $\zeta_{y}, 0 \leq \zeta_{x}, \zeta_{y} \leq 1$, and max- imum displacement $\delta r_{\max }$. The value of $\delta r_{\max }$ was adjusted during the simulations so that an average of $50 \%$ of the attempted moves was accepted. Its value was not, however, allowed to exceed $0.1 \sigma$. Moves which would result in an overlap of the particles were rejected.

As part of the constant $N p T$ simulations it is necessary to uniformly scale the system. ${ }^{32}$ A random change in the simulation box size $\delta L$ is considered where $\delta L$ is given by

$$
\delta L=(2 \zeta-1) \delta L_{\max },
$$

with random number $\xi, 0 \leq \xi \leq 1$, and maximum change in box length $\delta L_{\max }$. $\delta L_{\max }$ is independently adjusted to give an acceptance ratio of $50 \%$ for the area moves. The probability of accepting a random change from state $i$, at area $A(i)=L_{i}^{2}$, to state $j$, at area $A(j)=L_{j}^{2}=\left(L_{i}+\delta L\right)^{2}$, is given by $\min \left(1, P_{i j}\right)$ where

$$
\begin{aligned}
& P_{i j}=\exp \left(-\delta H_{i j} / k T\right), \\
& \delta H_{i j}=p \Delta A-N k T \ln [A(j) / A(i)] .
\end{aligned}
$$

Here $p$ is the two-dimensional or lateral pressure. Moves which would result in overlap were rejected. A scaling of the system was attempted once every complete Monte Carlo cycle (one attempted move per particle).

\section{B. Voronoi tesselation}

We have developed a method of keeping track of nearest and next-nearest neighbors, using the Voronoi tesselation of a system, which is applicable to highdensity systems and gives CPU time proportional to $N$.

For a set of $N$ seed points, $P_{i}(i=1, \ldots, N)$, in the Euclidean plane, $R^{2}$, the plane can be partitioned into $N$ regions $V_{N}\left(P_{i}\right)$, such that $V_{N}\left(P_{i}\right)$ is the locus of all points closer to $P_{i}$ than to any other point $P_{j}$. That is,

$$
V_{N}\left(P_{l}\right)=\bigcap_{j \neq i}\left\{P \in R^{2} \mid d\left(P, P_{i}\right)<d\left(P, P_{j}\right)\right\},
$$

where $d$ is the Euclidean distance. The planar skeleton $V_{N}$ formed by the boundaries of $V_{N}\left(P_{i}\right)(i=1, \ldots, N)$ is a planar straight-line graph and is called, among other things, the Voronoi diagram or tesselation (for example, ${ }^{33}$ Voronoi polygons, Wigner-Seitz cells, Dirichlet regions or tesselations, the cell model, the $S$ mosaic, Thiessen polygons, plant polygons, Wirkungsbereich, etc.) The edge between adjacent regions $V_{N}\left(P_{i}\right)$ and $V_{N}\left(P_{j}\right)$ is the perpendicular bisector between points $P_{i}$ and $P_{j}$.

We consider the Voronoi tesselation of the system of particles to be studied, with the centers of mass of the particles as the seed points of the tesselation. Periodic boundary conditions are applied to the system and the tesselation. This, in effect, puts the system on a toroidal surface. Euler's relation for a torus is $F-E+V=0$, where $F=N$ is the number of faces, $E$ is the number of edges, and $V$ is the number of vertices. The number of edges in the system is $E=3 N$ and the number of vertices is $V=2 N$. Also, the average number of edges around each seed point is identically 6 . For a system with open boundaries this applies only in the limit of large $N$. We establish a lookup table for the Voronoi edges. For each edge we store the adjacent points, or particles, its ver- 

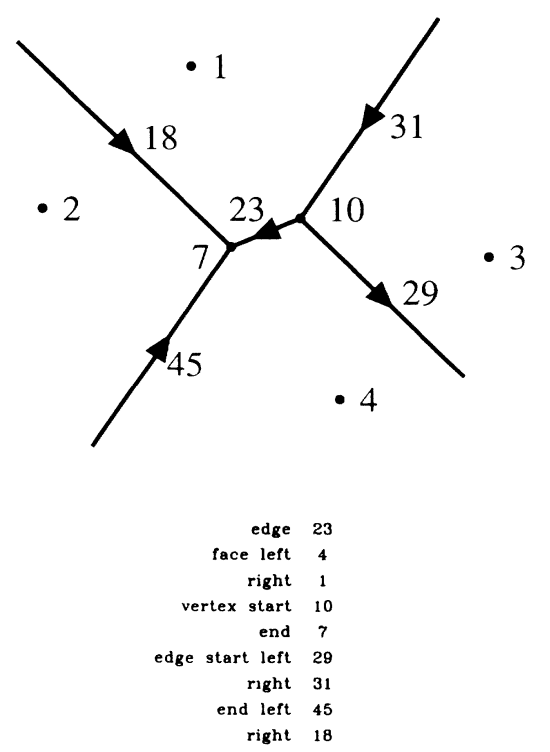

FIG. 1. A configuration of four particles and part of the Voronoi tesselation. The lookup-table entry for the central edge (23) is also shown.

tices, and the four adjoining edges, as shown in Fig. 1. From this table a list of neighbors for any particle can be determined as required. Two particles are neighbors if they have an edge in common. It is also possible to define next-nearest neighbors. Two particles are defined to be next-nearest neighbors if they are not neighbors but are connected by an edge. By connected we mean that one vertex of the edge is adjacent to one of the particles and the other vertex is adjacent to the other particle. For the example given in Fig. 1, particle 1 has neighbors 2, 3, and 4 , and particles 2 and 3 are next-nearest neighbors. The Delaunay triangulation, or dual of the Voronoi tesselation is the straight-line graph formed by connecting each pair of nearest neighbors.

\section{Initial configurations}

The initial configurations for the simulations were set up as described in Ref. 31 but with the addition of the Voronoi tesselation. Extreme low-density systems were first set up by placing $N$ points, at random, within the simulation cell, which was taken to be square. The Voronoi tesselation of this system, as described above, was then constructed according to the "divide-and-conquer" method described in Ref. 30 and outlined in the Appendix. Hard-core disks were allocated to the points. Each disk was given diameter $\sigma=d_{\text {min }}$, the minimum interparticle distance. The low-density systems were gradually compressed by performing Monte Carlo simulations, as described above, with a large applied pressure. This differed slightly from the previous method ${ }^{31}$ in which the system was contracted by an amount given solely by the minimum interparticle distance. Here the systems could expand or contract, but the large applied pressure would favor contraction. The systems were compressed until the required density was obtained. The loweset-density systems studied here were obtained in this way. Higher- density systems were obtained by compressing from a lower-density system. These systems were then equilibrated further.

\section{Updating the Voronoi tesselation dynamically}

When a particle is moved during the Monte Carlo simulation, as described in Sec. II A, the Voronoi edge list is not completely recalculated. If the particle moves are small (which is achieved by limiting the value of $\delta r_{\max }$ in the simulations) then the change in the Voronoi structure will only affect nearest and next-nearest neighbors. It is then possible to maintain the structure dynamically. There are three possible transformations of the Voronoi tesselation structure within a group of four particles. These are described below and illustrated in Fig. 2.

(a) The structure of that part of the Voronoi tesselation does not change, even though the coordinates of the vertices around the moved particle do change.

(b) The structure changes and a next-nearest neighbor becomes a nearest neighbor.

(c) The structure changes and a nearest neighbor becomes a next-nearest neighbor.

(a)

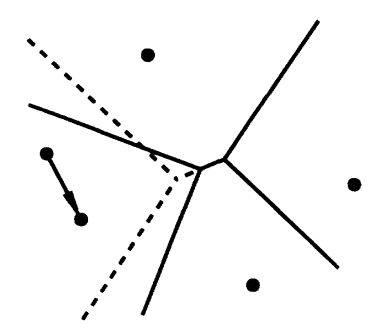

(b)
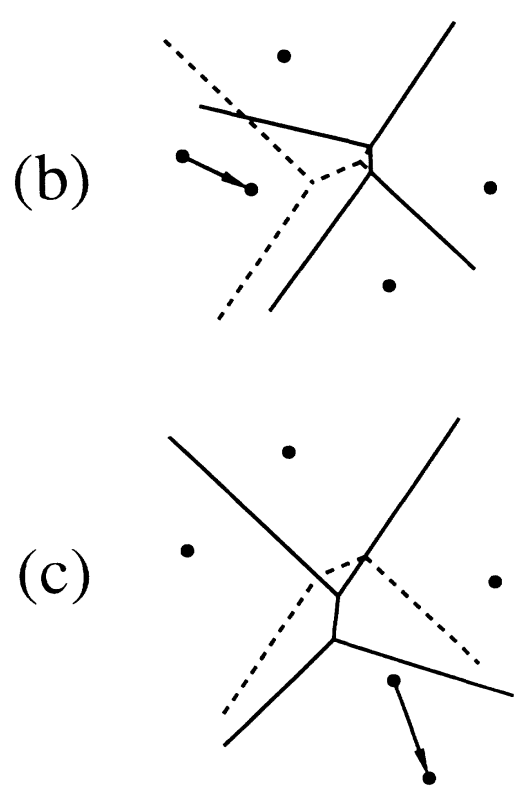

FIG. 2. Changes in the Voronoi tesselation structure brought about by moving a particle. The dashed and solid line indicate the initial and final structures, respectively. The three figures correspond to the situations described in the text. 
In each case, the numbers of edges and vertices in the tesselation are conserved. The only calculation that is required to update the tesselation is a recalculation of the coordinates of the vertices around the moved particle and, in cases (2) and (3), an updating of the edge lookup table. For a two-dimensional system with periodic boundary conditions each particle must have an average of six neighbors. Since the work involved in updating the tesselation depends only upon the number of neighbors a particle has, the total work involved in maintaining the tesselation must be linear in $N$. The computing time used is shown in Fig. 3 for systems of hard-core disks with periodic boundary conditions. Results are shown as a function of $N$. It can be seen that the computing time used for the Voronoi method is proportional to $N$. Simulations which use standard neighbor list methods ${ }^{32}$ require computing time which tends to increase linearly with $N$, but this does not include the overhead of updating the list, which is quadratic in $N$.

During the constant $N p T$ simulations the system area is changed as previously described. The area change is effected by expanding or contracting the whole system. The separation of any two points within the system is scaled by $\gamma$ where $\gamma^{2}=A(j) / A(i)$. The updating of the Voronoi tesselation thus only requires a scaling of the vertex coordinates: the structure is invariant.

\section{Analysis of the Voronoi tesselation}

There are many distribution functions that can be obtained from the Voronoi tesselation. The most obvious is the number of particles with $n$ neighbors, $p(n)$. The fractional number of defects in the system can be defined as

$$
N_{\mathrm{def}}=\frac{\sum_{n \neq 6} p(n)}{\sum_{n} p(n)} .
$$

Another distribution which has received some attention is $m_{n}$, the average number of neighbors of particles

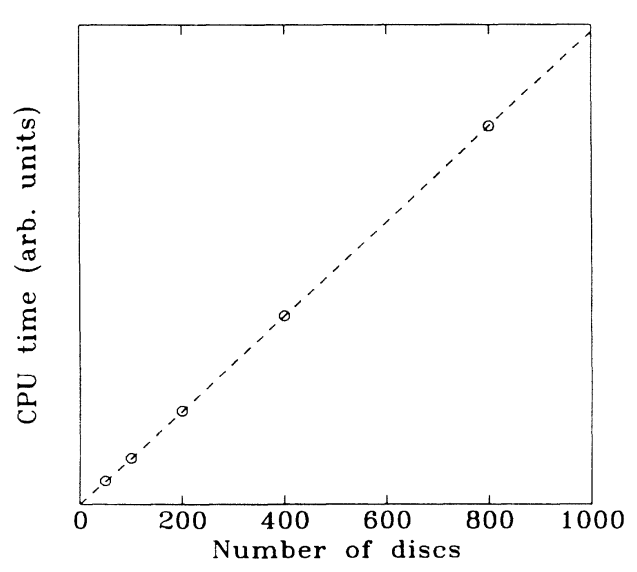

FIG. 3. The computing time used as a function of the number of particles in the system. The times for four runs, each of 100 cycles, are shown for each value of $N$. The data are for the method presented here with $\eta \rightarrow 0$. The dashed line is a linear least-squares fit to the data. which are neighbors to a particle with $n$ neighbors (see Refs. 34-38 and references therein). It has been fairly well established, empirically, that this function satisfies the equation

$$
m_{n}=6-a+(6 a+c) / n,
$$

where $a$ and $c$ are numerical parameters. Within the accuracy of the data, $c$ is equal to the variance of the distribution $p(n)$. It is also suggested that $a \propto \alpha$ where

$$
\alpha=\left(s_{x}-s_{y}\right) /\left(s_{x}+s_{y}\right)
$$

is the differential longitudinal or metrical dispersion. $\left(s_{x} \bar{x}\right)$ and $\left(s_{y} \bar{y}\right)$ are the standard deviations of $x$ and $y$, the lengths of the edges in the tesselation and its dual, respectively. Data generated from the studies described here have been used to confirm the form of Eq. (6) for Voronoi tesselations generated from hard-disk systems. It is also found that $a$ is not directly proportional to $\alpha$, as defined in Eq. (7), for these systems. These results are planned to be reported in a subsequent paper. ${ }^{39}$

Little work has been done in studying the shape of the distribution of edge lengths of the Voronoi tesselation, $p(d)$. In studying the Voronoi tesselations of hard-disk systems, we have found that the total distribution function $p(d / \sigma)$ can be fitted by the sum of two Gaussian functions, as described below. The fits obtained are good at all densities and the fitting parameters are found to reflect structural properties of the disk systems. A very good fit is also obtained in the low-density limit. Figure 4 shows the distribution of edge lengths for random systems of points, including data from Ref. 40. The natural tesselations looked at by $\mathrm{Aboav}^{41}$ are not Voronoi tesselations in the sense that they cannot be generated from a set of seed points by Eq. (4). The true Voronoi tesselations, refered to in Aboav's paper, all produced edgelength distribution functions which appear similar to those shown in Fig. 4, though the relatively small number of edges sampled is apparent in the data.

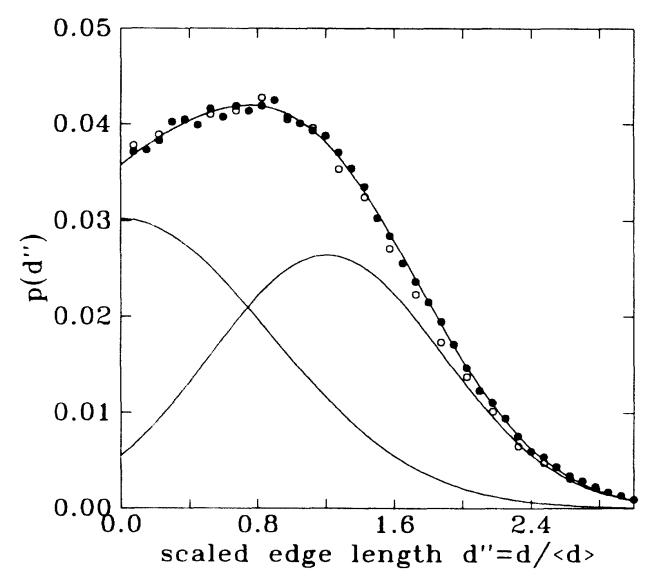

FIG. 4. The data $(O)$ give the distribution of Voronoi edge lengths, $p\left(d^{\prime \prime}\right)$, for random distributions of points, averaged over a total of 45000 edges. The data (O) are taken from Ref. 40. The solid lines give the least-squares fit of the function $g\left(d^{\prime \prime}\right)$ of Eq. (8), and its two component Gaussians, fitted to the data $(\bigcirc)$. 


\section{Fitting $p(d / \sigma)$}

The distribution functions $p(d / \sigma)$ obtained from the Monte Carlo simulations were fitted by a function of the form

$$
\begin{aligned}
g\left(d^{\prime}\right)= & \frac{1}{\sqrt{\pi} \omega_{1}} I_{1} e^{-\left(d^{\prime}-Q_{1}\right)^{2} / \omega_{1}^{2}} \\
& +\frac{1}{\sqrt{\pi} \omega_{2}} I_{2} e^{-\left(d^{\prime}-Q_{2}\right)^{2} / \omega_{2}^{2}}
\end{aligned}
$$

where $d^{\prime}=d / \sigma$. The parameters $Q_{1}, Q_{2}, W_{1}=\left(\omega_{1} \ln 2\right)$, and $W_{2}=\left(\omega_{2} \ln 2\right)$ represent the means and widths of the two Gaussians in units of $\sigma . I_{1}$ and $I_{2}$ give the areas under each of the two Gaussians. Values for the parameters were obtained by a least-squares fit to the Monte Carlo data. The term "main Gaussian" will refer to the Gaussian which is mainly responsible for the peak in the distribution function $p\left(d^{\prime}\right)$ and its fit parameters will be denoted by the subscript 1 . The other Gaussian, the "secondary Gaussian," will be denoted by the subscript 2 .

We emphasize that the functional form of $g\left(d^{\prime}\right)$ has no physical basis. The fit used is found to be a useful tool which we will show provides an excellent way of looking at the structure of the systems.

\section{RESULTS}

Simulations were performed on systems of 102 and 408 hard-disks at pressures corresponding to reduced pressures, $\varphi=p / \rho k T$, of between 4.2 and 25.0 (packing fractions ranging from 0.49 to 0.82 ). For the 102 disk systems a total of 20000 cycles was considered at each pressure. The first 10000 of these were used for equilibration purposes and were discarded. The remaining 10000 were used for calculating equilibrium, thermodynamical properties of the systems. For the 408 disk systems 10000 cycles were considered at each pressure, of which 6000 were discarded and 4000 were used for system properties. Statistics were accumulated every ten cycles for both system sizes. Since there were few differences between the results obtained from the 102 disk systems as compared with the 408 disk systems we present only the 408 disk results, except where the differences are significant. Snapshots of the Voronoi tesselations for some of these systems are shown in Figs. 5(a)-5(h). At the lowest densities the structure is definitely that of a disordered fluid. As the pressure, and hence density, is increased the degree of order in the systems increases. There are indications of a transition to a more ordered phase between packing fractions in the liquid phase of around $\eta_{\mathrm{lq}}=0.69$ and in the solid phase of $\eta_{\text {sol }}=0.73$. This corresponds to the transition region found by Alder and Wainwright ${ }^{1}$ for which $\eta_{\text {liq }}=0.691$ and $\eta_{\text {sol }}=0.716$. Within this region there are disordered domains of ordered disks. The size of the domains increases with density until the systems exhibit structure indicative of a crystal. It should be noted that the structure is not that of a commensurate crystal since the systems contain defects. Preliminary studies on systems of 102 disks produced some high-density structures which had a defect in the crystal structure and others which gave a defectless crystal structure commensurate with the simulation box. The effect of the presence of defects on the results will be discussed later.

\section{A. Distribution of neighbors}

The distributions of neighbors, $p(n)$, are shown in Fig. 6 for several of the systems. It can be seen that the most probable number of neighbors is 6 for all densities shown. The width of the distribution decreases with increasing density. The fractional number of defects $N_{\text {def }}$, given by Eq. (5), is shown in Fig. 7. Although $N_{\text {def }}$ decreases from the disordered phase to the ordered phase, it does not go identically to zero. This is due to the presence of defects

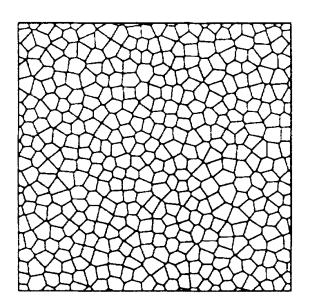

(a)

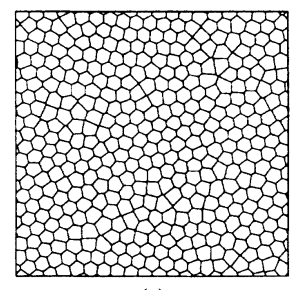

(e)

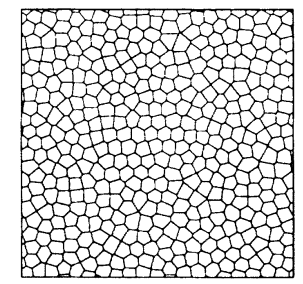

(b)

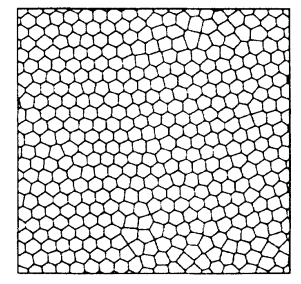

(f)

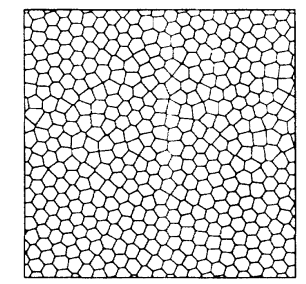

(c)

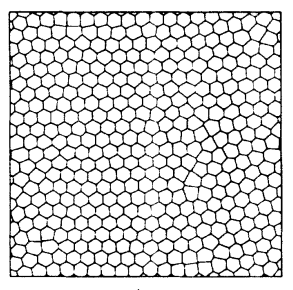

(g)

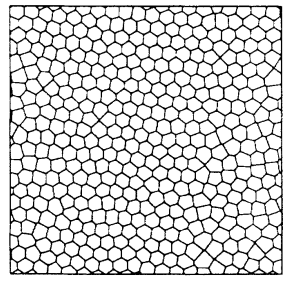

(d)

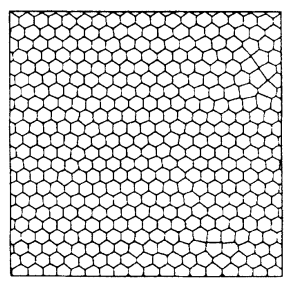

(h)

FIG. 5. Snapshots of configurations of 408 hard disks at pressures $p / k T$ of (a) $2.61 \sigma^{-2}$, (b) $6.62 \sigma^{-2}$, (c) $9.50 \sigma^{-2}$, (d) $10.3 \sigma^{-2}$, (e) $10.9 \sigma^{-2}$, (f) $11.8 \sigma^{-2}$, (g) $14.1 \sigma^{-2}$, (h) $20.3 \sigma^{-2}$. The packing fractions $\eta$ of the configurations shown are (a) 0.492 , (b) 0.647 , (c) 0.703 , (d) 0.725 , (e) 0.727 , (f) $0.734,(\mathrm{~g}) 0.756$, (h) 0.797 . The systems are scaled to the same box size and only the Voronoi tesselations of the systems are shown. 


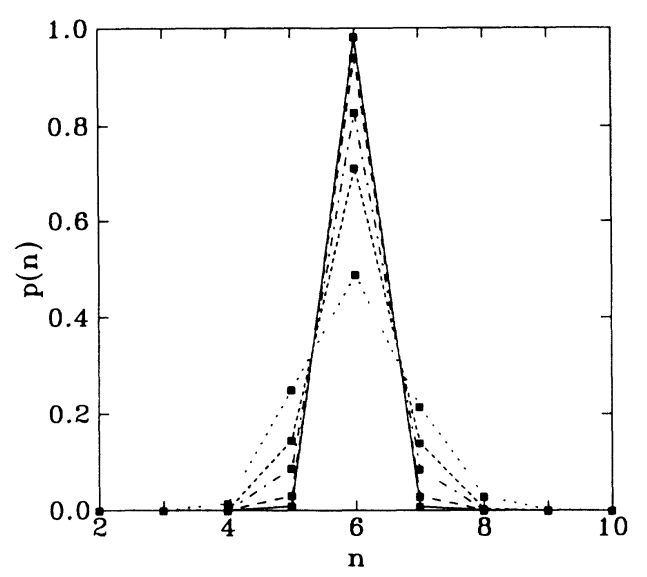

FIG. 6. The distributions of neighbors $p(n)$ for systems at pressures $p / k T$ of $(. .) .2.61 \sigma^{-2},(--) 8.05 \sigma^{-2},(-.-\cdot-\cdot)$ $10.0 \sigma^{-2},(--\longrightarrow) 11.8 \sigma^{-2},(-) 26.2 \sigma^{-2}$.

within the crystal-like structure. If the systems were to form commensurate crystals then each disk would have six neighbors giving $N_{\text {def }}=0$. The exact position of the transition region is not clear from the data in Fig. 7.

\section{B. Voronoi edge-length distribution}

The distribution functions $p\left(d^{\prime}\right)$ are shown in Fig. 8 for several of the systems studied. The points give the Monte Carlo data and the solid lines give $g\left(d^{\prime}\right)$ and also each of the two Gaussian components of $g\left(d^{\prime}\right)$. The closeness of the fits at all densities within the fluid and solid phases suggests that the functional form of $g\left(d^{\prime}\right)$ is reasonable. Only for the systems around the transition region, for which $10 \lesssim \varphi \lesssim 12$, is there any deviation of the fit from the data. This will be discussed further below.

At low densities the distributions are broad and there is a significant contribution from edges tending to zero length. This is best illustrated by the distribution shown

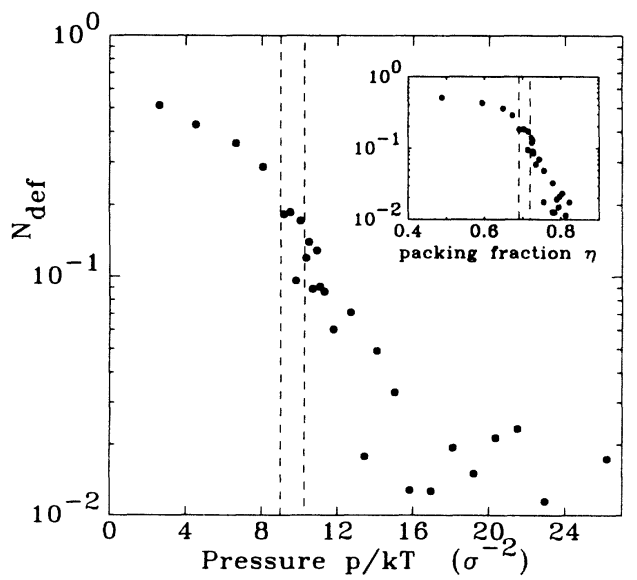

FIG. 7. The fractional number of defects in the system $N_{\text {def }}$ as a function of pressure for the 408 disk systems studied. The inset gives the same data as a function of packing fraction. The dashed lines indicate the transition region.

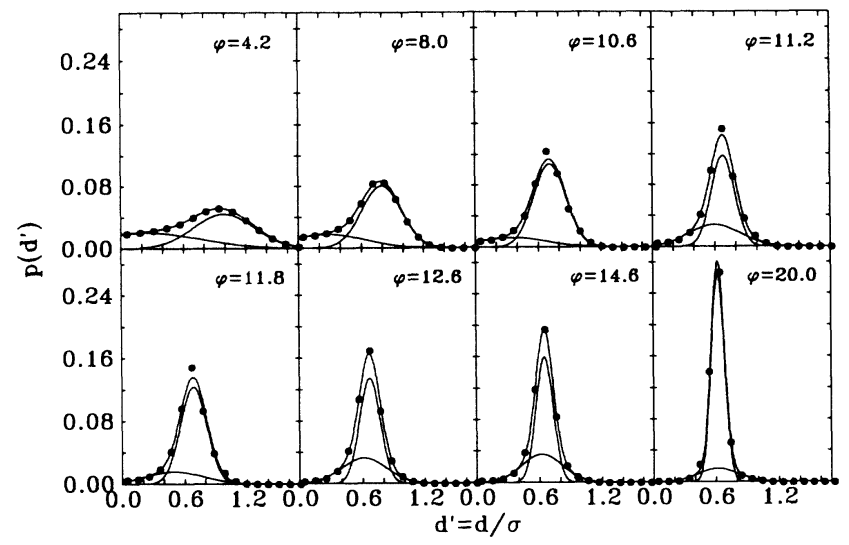

FIG. 8. The distributions of Voronoi edge lengths, $p\left(d^{\prime}\right)$, for systems with the given reduced pressures. The solid lines give the least-squares fits $g\left(d^{\prime}\right)$ of Eq. (8) and their Gaussian components.

in Fig. 4, which is for the extreme low-density, random case. Note that the equivalent disk diameter $\sigma_{r} \rightarrow 0$ as $\eta \rightarrow 0$, so the edge lengths are scaled with respect to the average edge length $\langle d\rangle$. Here the secondary Gaussian, which is centered around $d^{\prime \prime}=d /\langle d\rangle \gtrsim 0$, has a greater maximum value than that of the main Gaussian. For the lowest-density systems studied here, the secondary Gaussian is still centered around $d^{\prime} \gtrsim 0$ but its intensity is greatly reduced. As the density is increased the mean moves to longer edge lengths and its intensity remains roughly constant. The main Gaussian is seen to shift to slightly shorter lengths with increasing density. Its intensity increases and its width decreases.

The transition region appears to have little effect upon the distribution, though the fits obtained are not as good as for the fluid and solid phases. The maximum point of the distribution is always underestimated even though estimates for the adjacent points are reasonable. Within the transition region the mean of the secondary Gaussian fluctuates from its low-density maximum value of about 0.4 to a value of about 0.6 , which is only slightly below that of the main Gaussian. This higher value is accompanied by a greater intensity. Accordingly, the intensity of the main Gaussian is reduced, though its position appears unaffected.

At high densities, that is, for systems which exhibit crystal-like structure, the means of the two Gaussians are almost coincidental. For densities just above the transition region the mean of the secondary Gaussian is at slightly shorter edge lengths than that of the main Gaussian. For the extreme high-density systems their positions are reversed. The main Gaussian is, by definition, much more intense than the secondary Gaussian. It is also much sharper, the secondary curve being very broad.

The question of whether the functional form for the high-density distributions should be the sum of two Gaussians, and not just a single Gaussian, can be answered by comparing the distributions for the lowestdensity solid systems and the highest-density solid systems. As was mentioned above, the relative positions of the two Gaussians change as the density is increased. 
This would seem to be not just an artifact of the fitting procedure. If the shapes of the tails of the distributions are compared then the distributions are seen to be asymmetrical. This asymmetry is reflected in the fits obtained, though the fits for the longer edge-length tails are better than those for the shorter edge-length tails.

A similar asymmetry can also be seen for the smaller, 102 disk, systems. Figure 9 shows the distributions for two systems each with the same reduced pressure of 19.0. The two systems differ in that one has condensed to a system with a defect structure and the other has condensed to a commensurate crystal. Whereas the distribution $p\left(d^{\prime}\right)$ for the defect structure is symmetrical and the two Gaussian means are coincidental, the distribution for the commensurate crystal is not. This is most apparent if a comparison is again made of the tails of the distributions. The short edge-length tails are very similar but the long edge-length tail of the commensurate crystal data drops off much more sharply than that of the defect structure data. Its intensity is also considerably greater. This difference in symmetry is consistent through all of the 102 disk systems studied, though only one pair of distributions is shown here.

Consideration of crystalline structures would lead one to expect fewer short edges and fewer long edges. This would give a sharper, more intense distribution. The long edge-length tail of the observed distributions supports this but the short edge-length tail does not. The total effect would be consistent, though, if there were strain in the system. The presence of a uniaxial strain would tend to increase the disk separations in one particular direction and decrease the normal components of other separations. This would have the effect of reducing the lengths of edges lying perpendicular to that direction and marginally increasing the lengths of the other edges in the system. The effect on the long edge-length tail of the distribution would be small. The short edge-length tail would, however, increase significantly, thus canceling the effect of the more regular crystalline structure. The distributions observed are, therefore, consistent with a

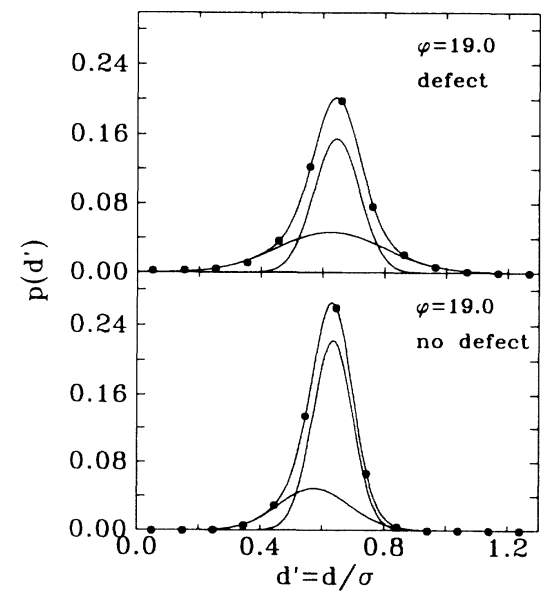

FIG. 9. The distribution functions $p\left(d^{\prime}\right)$ for two 102 disk systems with reduced pressures of 19.0. The upper distribution is for a system with defects. The lower distribution is for a system with no defects.

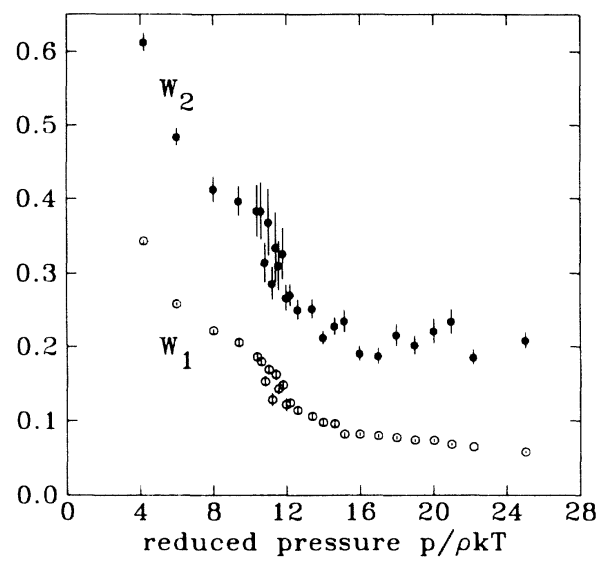

FIG. 10. The fit parameters $W_{1}(\bigcirc)$ and $W_{2}(\bigcirc)$ as a function of reduced pressure for 408 disk systems.

strained crystalline structure.

Figure 10 shows the fitting parameters $W_{1}$ and $W_{2}$ as functions of reduced pressure for the 408 disk systems. The widths of both Gaussians are seen to decrease with increasing pressure. That of the secondary Gaussian does increase again at the highest densities. In each case there is a suggestion of a discontinuity across the phase transition region. This is accompanied, however, by increased uncertainty in the fits, indicated by larger error bars.

Figure 11 shows the fitting parameters $I_{1}$ and $I_{2}$, again for the 408 disk systems. The sum $I=I_{1}+I_{2}$ is shown in the inset. Apart from for the low-density systems, it can be seen that $I$ is constant. This is reassuring since it represents $\int g\left(d^{\prime}\right) d d^{\prime}$ and $g\left(d^{\prime}\right)$ is being fitted to normalized data. The deviation at low density is because a significant part of the secondary Gaussian corresponds to nonphysical (negative) values of $d^{\prime}$. It is clearer here than in Fig. 10 that there is some sort of discontinuity across the phase transition region. In both the liquid and solid phases the proportions of edges which contribute to the main Gaussian increase with increasing density.

The fits to the means of the two Gaussians are shown

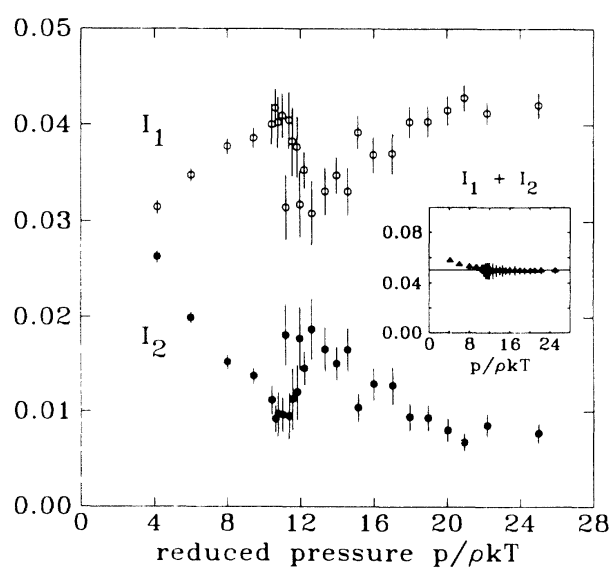

FIG. 11. The fit parameters $I_{1}(O)$ and $I_{2}(O)$ as a function of reduced pressure for 408 disk systems. The inset gives the sum $I_{1}+I_{2}$. 
in Fig. 12 for the 408 disk systems. The inset gives the data for the 102 disk systems. With the exception of some of the 102 disk data, which will be discussed below, both means tend to approximately the same asymptote at high density. As observed above, the value of $Q_{2}$ is still increasing slightly, whereas that of $Q_{1}$ is decreasing. The solid line gives the edge length that would be obtained is the cells of the Voronoi tesselations of the systems were regular hexagons. The mean of the main Gaussian is within statistical error of this curve at high densities. The 102 disk data is very similar to that of the 408 disk systems. The data for $Q_{1}$ has the same asymptote. The data for $Q_{2}$, however, is split between two, seemingly parallel, asymptotes. If the systems are grouped according to whether or not their crystal-like structure contains defects, then it is found that, without exception, at high densities, the data for the systems with defects has its asymptote close to that for $Q_{1}$ and the data for the commensurate crystal systems has the parallel, lower asymptote. This is consistent with the observed symmetry of the distributions.

The effect is not simply a system size effect since a single defect structure in the 102 disk systems would affect proportionately more Voronoi edges than in the 408 disk systems. If the lower asymptote were the true asymptote for infinite system sizes and the upper one a deviation due to the relatively high proportion of defects in the systems then it should be expected that the data for the 408 disk systems would tend to the lower asymptote, which is not the case. Since the commensurate crystal structures obtained cannot be truly commensurate with the square simulation cell used, it is likely that the shift in the asymptote is indeed caused by a strain of the structure, as discussed earlier. If there was little or no strain in the 102 disk systems then the 408 disk systems, which have exactly four times the number of disks, would be expected to condense to commensurate structures similar to those of the 102 disk systems. Instead they condense to a structure representative of a triangular lattice containing

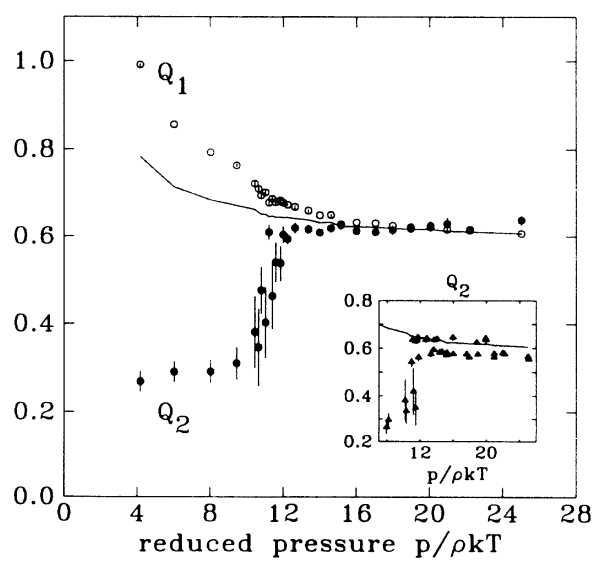

FIG. 12. The fit parameters $Q_{1}(\bigcirc)$ and $Q_{2}(\bigcirc)$ as a function of reduced pressure for 408 disk systems. The error bars for $Q_{1}$ are within the open circles. The solid line gives the edge length that would be obtained if each Voronoi cell was a perfect hexagon. The inset gives $Q_{2}$ for the 102 disk systems.
22 rows each of 19 disks, but with half a row (10 disks) missing.

There is a very sharp jump in the value of $Q_{2}$ across the transition region. The observed fluctuations within this region are reflected by large error bars in the values of $Q_{2}$. It should be noted that the error bars for the data within the liquid phase are small by comparison, though not as small as in the solid phase. In contrast, the error bars for $Q_{1}$ are contained within the points drawn, at all densities.

In the fluid phase the secondary Gaussian corresponds to a population of short edges. In the solid phase the structure is highly ordered and there are few short edges. The short edges that are to be found in these systems can be associated with regions of lower order, or defects. The secondary Gaussian does not, therefore, correspond only to the presence of short edges but it does allow a better fit to the asymmetric tails of the total distribution. The effects seen with the 102 disk systems support this.

\section{Thermodynamic properties}

The $p$ - $A$ isotherm for the 408 disk systems is shown in Fig. 13. The dashed line gives the corresponding SPT isotherm $^{9}$

$$
\frac{p}{\rho k T}=\frac{1}{(1-\eta)^{2}},
$$

where $\eta=(\pi / 4) \rho \sigma^{2}$ is the packing fraction of the system. The short-dashed line is a guide for the eye only. It consists of the SPT data in the fluid phase and the crystalphase isotherm of Andrews ${ }^{16}$ in the solid phase. The joining line is a linear, least-squares fit, to the data across the transition region. Data from other studies are shown for comparison including the van der Waals-like loop from Alder and Wainwright. ${ }^{1}$ The transition region is apparent, though its solid phase limit cannot be isolated as easily as it could with the Voronoi edge-length distribution described above. Even though the data for the first

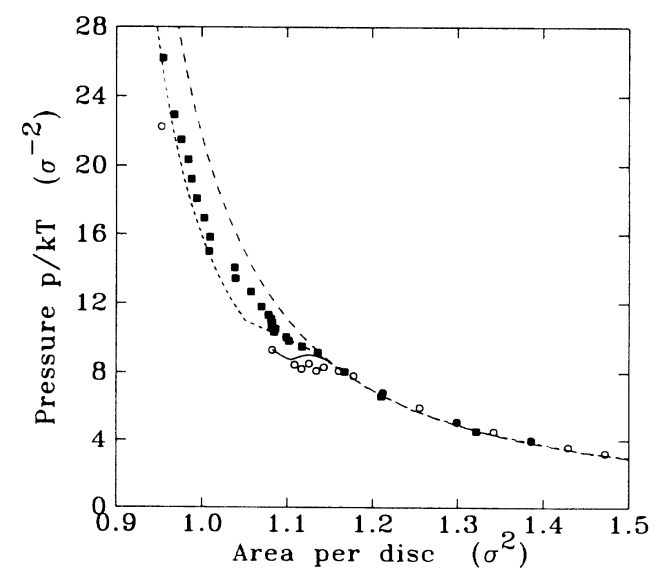

FIG. 13. Pressure as a function of area per disk for the 408 disk systems. (ם). The short-dashed line is described in the text. The dashed line is the SPT data from Eq. (9). The solid line gives the van der Waals-like loop from Ref. 1. The remaining data are taken from Ref. $2(0)$ and Ref. $20(\bigcirc)$. 
2000 cycles considered were coincident with those for the second 2000 cycles, there is scatter in the data just within the solid phase. This indicates that the systems were not fully at equilibrium, and highlights the difficulty in obtaining equilibrium thermodynamic properties around phase transitions. Even though the highest-density systems do not exhibit perfect crystalline order, the results for these systems compare well with the isotherm of Andrews. The agreement of the data with scaled particle theory in the liquid phase is good.

\section{SUMMARY AND CONCLUSIONS}

We have presented in this paper a technique, based on the Voronoi tesselation, for detecting structural transitions in two-dimensional condensed systems. The technique was tested on a two-dimensional hard-disk system. The edge-length distribution of the Voronoi tesselation, determined as a function of external pressure, was used to calculate the quantity $Q_{2}$ of Eq. (8). $Q_{2}$ reflects the degree of structural order within the system and provides a strong signal for the liquid-solid transition of the harddisk system. This signal is superior to that from the pressure-area isotherm or from the defect densities.

Dynamical updating of the Voronoi tesselation during the Monte Carlo simulation allows for properties of the tesselation to be available for use during the entire simulation run without having to recalculate the tesselation. For example, the tesselation provides easy access to nearest and next-nearest neighbor pairs. Also, since the Voronoi edge lengths correspond to the interfacial lengths between nearest neighbors, these lengths can be used in the calculation of contact interactions between components of complex systems.

We have applied these techniques to binary mixtures of disks in which the concentration is allowed to vary with density and pressure according to preset degeneracies. These results are planned to be presented in a subsequent paper. $^{42}$

\section{ACKNOWLEDGMENTS}

This work was supported by the Natural Sciences and Engineering Research Council of Canada, by Fonds pour la formation de Chercheurs et l'Aide á la Recherche du Quebec, and by the Danish Natural Science Research Council under Grant No. J.nr.5.21.99.71. Diane Fraser's work in Denmark was supported by the Danish Natural Science Research Council under Grant No. J.nr.11-7785 and Martin Zuckermann's work in Denmark was supported by the Danish Research Academy under Grant No. J.nr.F890095. Stimulating discussions with Søren Toxværd and Eigil Præstgaard on two-dimensional melting are gratefully acknowledged.

\section{APPENDIX: CONSTRUCTING THE VORONOI TESSELATION FROM A SET OF SEED POINTS}

Since the Voronoi tesselation for a system is only calculated once and then maintained dynamically throughout the Monte Carlo simulations, the time required to compute the tesselation was not a factor in deciding which algorithm to use. The main requirement is that the data structure describing the tesselation allows easy access to the information required, namely, nearest and next-nearest neighbors. Despite this, the algorithm used is fast and can be shown to run in $N \ln N$ time. This compares with $N^{2}$ time for a neighbor search algorithm.

The Voronoi tesselation $V_{N}$ for a given set $P$ of $N$ seed points was set up following the divide-and-conquer method described by Preperata and Shamos. ${ }^{30}$ Consider two linearly separated subsets of $P, P_{i}^{(1)}\left(i=1, \ldots, N_{1}\right)$ and $P_{i}^{(2)}\left(i=1, \ldots, N_{2}\right)$, such that

$$
\begin{aligned}
& P_{i}=P_{l}^{(1)} \cup P_{i}^{(2)}, \\
& N=N_{1}+N_{2} .
\end{aligned}
$$

Without loss of generality, assume that $P_{i}^{(1)}$ and $P_{i}^{(2)}$ are separated by a vertical line. Let $V_{N_{1}}$ and $V_{N_{2}}$ denote the Voronoi tesselations generated by the subsets $P_{i}^{(1)}$ and $P_{i}^{(2)}$, respectively. If the tesselations $V_{N_{1}}$ and $V_{N_{2}}$ are known then they can be "merged" to give $V_{N}$ by constructing the dividing chain $\sigma$. To construct $\sigma$ the convex hull of each subset is required. The first and last segments of $\sigma$ are the perpendicular bisectors of the supporting segments of the convex hulls of the two subsets, as shown in Fig. 14. Once the first segment is determined from the convex hulls, segments are added to $\sigma$ as it intersects with successive edges of the two tesselations. See Fig. 15. This continues until the last segment is added. $\sigma$ thus proceeds by an irregular, zigzag path between the two tesselations, joining them as it goes. It should be noted that the convex hull of $P_{i}$ can be obtained easily from the convex hulls of $P_{i}^{(1)}$ and $P_{i}^{(2)}$ and the supporting segments.

Since $V_{2}$ and $V_{3}$ are trivial, the method can be applied as follows.

Step 1. Divide $P_{i}$ into two subsets, $P_{i}^{(1)}$ and $P_{i}^{(2)}$ of approximately equal sizes, $N_{1}$ and $N_{2}$.

Step 2. Construct $V_{N_{1}}$ and $V_{N_{2}}$ recursively.

Step 3. Merge $V_{N_{1}}$ and $V_{N_{2}}$ to obtain $V_{N}$.

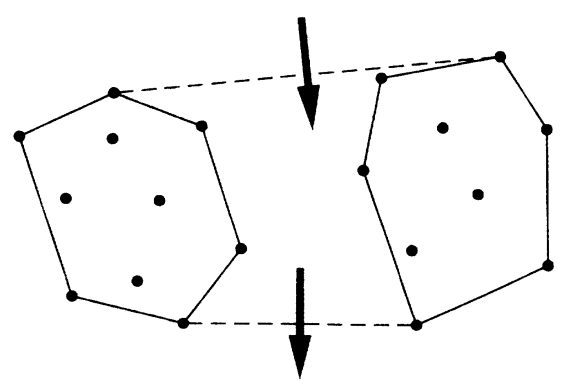

FIG. 14. The dashed lines are the supporting segments of the two convex hulls, which are given by the solid lines. The first and last segments of $\sigma$, indicated by the arrows, are the perpendicular bisectors of these supporting segments. 


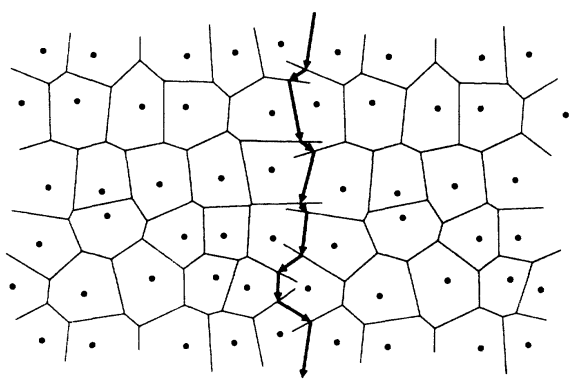

FIG. 15. Two tesselations $V_{N_{1}}$ and $V_{N_{2}}$ are merged by constructing the dividing chain $\sigma$, shown here by the heavy arrows. Only subsections of $V_{N_{1}}$ and $V_{N_{2}}$ are illustrated.

Here we wish to apply periodic boundary conditions so that the system lies on a toroidal surface. This is a twostage process. Assume that we have constructed the open boundary Voronoi tesselation. Shift the $x$ coordinates of the system, while applying periodic boundary conditions, so that the open, vertical edges of the tesselation lie down the center of the simulation box. The left half of the box will contain the right edge of the tesselation and the right half of the box will contain the left edge. The two edges can be joined, as above, by assuming that the two halves correspond to different subsets $P_{i}^{(1)}$ and $P_{i}^{(2)}$. The system now corresponds to the surface of a cylinder. We join the two remaining open edges in a similar manner. For convenience, the system is rotated before being shifted so that the open edges are again vertical. Since the edges are continuous we have to choose a point of entry for $\sigma$. We consider the extreme right-hand point of the right edge (left half of the box) and the extreme left-hand point of the left edge (right half of the box). The first segment of $\sigma$ is taken to be the perpendicular bisector of the shortest line joining these two points. $\sigma$ is constructed as above, the last segment coinciding with the first. The two ends of $\sigma$ are joined and the last segment is discarded.

As well as constructing the Voronoi tesselation itself, we have to construct $E$, the edge list, or lookup table of edges, described in Sec. II B. Since most of the information stored in $E$ is required during the construction of $\sigma$ it is most convenient to update $E$ as each segment is added to $\sigma$. Once the ends of the final $\sigma$ have been joined then $E$ is also complete.
${ }^{1}$ B. J. Alder and T. E. Wainwright, Phys. Rev. 127, 359 (1962)

${ }^{2}$ W. G. Hoover and B. J. Alder, J. Chem. Phys. 46, 686 (1967).

${ }^{3}$ W. W. Wood, J. Chem. Phys. 52, 729 (1970).

${ }^{4}$ N. Metropolis, A. W. Rosenbluth, M. N. Rosenbluth, A. H.

Teller, and E. Teller, J. Chem. Phys. 21, 1087 (1953).

${ }^{5}$ K. J. Strandburg, Rev. Mod. Phys. 60, 161 (1988).

${ }^{6}$ W. W. Wood, J. Chem. Phys. 48, 415 (1968).

${ }^{7}$ D. G. Chae, F. H. Ree, and T. Ree, J. Chem. Phys. 50, 1581 (1969).

${ }^{8}$ J. P. Hansen I. R. MacDonald, Theory of Simple Liquids (Academic, New York, 1976).

${ }^{9}$ E. Helfand, H. L. Frisch, and J. L. Lebowitz, J. Chem. Phys. 34, 1037 (1961).

${ }^{10}$ M. A. Cotter and F. H. Stillinger, J. Chem. Phys. 57, 3356 (1972).

${ }^{11}$ D. Henderson, Mol. Phys. 30, 971 (1975).

${ }^{12}$ D. Henderson, Mol. Phys. 34, 301 (1977).

${ }^{13}$ B. J. Alder and T. E. Wainwright, J. Chem. Phys. 31, 459 (1959).

${ }^{14}$ F. H. Ree and W. G. Hoover, J. Chem. Phys. 40, 939 (1964).

${ }^{15}$ L. V. Woodcock, J. Chem. Soc. Faraday Trans. II 72, 731 (1976).

${ }^{16}$ F. C. Andrews, J. Chem. Phys. 64, 1941 (1976).

${ }^{17}$ F. H. Ree and W. G. Hoover, J. Chem. Phys. 46, 4181 (1967).

${ }^{18}$ B. J. Alder, W. G. Hoover, and D. A. Young, J. Chem. Phys. 49, 3688 (1968).

${ }^{19}$ K. W. Kratky, J. Chem. Phys. 69, 2251 (1978).

${ }^{20}$ J. J. Erpenbeck and M. Luban, Phys. Rev. A 32, 2920 (1985).
${ }^{21}$ J. Vieillard-Baron, J. Chem. Phys. 56, 4729 (1972).

${ }^{22}$ I. Nezbeda, T. Boublik, and O. Trnka, Czech. J. Phys. B 25, 119 (1975).

${ }^{23}$ D. Frenkel and R. Eppenga, Phys. Rev. A 31, 1776 (1985).

${ }^{24}$ J. Talbot and D. J. Tildesley, J. Chem. Phys. 83, 6419 (1985).

${ }^{25}$ G. Lasher, J. Chem. Phys. 53, 4141 (1970).

${ }^{26}$ T. Boublík, Mol. Phys. 29, 421 (1975).

${ }^{27}$ T. Boublik, Mol. Phys. 63, 685 (1988).

${ }^{28}$ D. A. Ward and F. Lado, Mol. Phys. 63, 623 (1988).

${ }^{29}$ D. A. Ward and F. Lado, Mol. Phys. 64, 1185 (1988).

${ }^{30}$ F. P. Preparata and M. I. Shamos, Computational Geometry, An Introduction (Springer-Verlag, New York, 1985).

${ }^{31}$ D. P. Fraser, R. W. Chantrell, D. Melville, and D. J. Tildesley, Liq. Cryst. 3, 423 (1988).

${ }^{32}$ M. P. Allen and D. J. Tildesley, Computer Simulation of Liquids (Oxford University Press, Oxford, 1987).

${ }^{33}$ D. Weaire and N. Rivier, Contemp. Phys. 25, 59 (1984).

${ }^{34}$ D. Weaire and J. P. Kermode, Philos. Mag. B 50, 379 (1984).

${ }^{35}$ N. Rivier, Philos. Mag. B 52, 795 (1985).

${ }^{36}$ C. J. Lambert and D. Weaire, Philos. Mag. 47, 445 (1983).

${ }^{37}$ D. A. Aboav, Metallography 18, 129 (1985).

${ }^{38}$ B. N. Boots, Metallography 18, 301 (1985).

${ }^{39}$ D. P. Fraser (unpublished).

${ }^{40}$ B. N. Boots, Metallography 20, 231 (1987).

${ }^{41}$ D. A. Aboav, Metallography 17, 383 (1984).

${ }^{42}$ D. P. Fraser, M. J. Zuckermann, and O. G. Mouritsen (unpublished). 